\title{
Un análisis científico-tecnológico de la investigación-acción
}

JULIO VERA VILA

Universidad de Málaga

\begin{abstract}
SUMMARY.-In this paper, the author studies the action-research in relation to scientifical and technological research. Usually both have been shown as two proposals set against each other.

The author recognizes the important contribution done by the action-research, how usefull are its proposals about qualitative methods and teacher's rol as an intellectual, who takes part in the design of his practice and thinks critically about the process. But from his point of view there are reasons in order to see the accion-research as a scientific-technological pattern. So, education should be looked as a technological practice based on a scientific theory.
\end{abstract}

\section{INTRODUCCIÓN}

La investigación-acción ha irrumpido con fuerza en el ámbito de la Pedagogía, de modo que el número de trabajos referidos a la misma crece incesantemente. Este crecimiento y su rápida aceptación entre los colectivos dedicados a la educación, nos obliga a reflexionar acerca de sus propuestas y de su contribución a la comprensión y optimización de los procesos educativos, a fin de aprovechar de este modelo todo cuanto contribuya a conocer y mejorar la acción educativa, sea cual sea el ámbito sobre el que ésta se ejecute.

En concreto, atrae mi atención el hecho de que la investigación-acción está siendo presentada como alternativa al modo de proceder tecnológico en la educación. Muchos autores han volcado una parte de su esfuerzo explicativo en confrontar esta modalidad de investigación socio-educativa con la científico-tecnológica, hasta el punto de parecer no sólo como diferentes sino, incluso, contrapuestas. Si llevamos algunos de los argumentos empleados a su extremo habría que decir que incluso lo tecnológico es obstáculo para la que ahora se presenta como la modalidad de investigación que mejor se adapta a las características del objeto de estudio: la educación o lo socio-educativo, en general.

Por lo tanto, quisiera centrar estas reflexiones en este punto: que desde los promotores de la investigación-acción, ésta representa una forma de conocimiento y de acción diferente de la racionalidad tecnológica, superadora de ésta, aunque no absolutamente excluyente. El grado de exclusión varía de unos autores a otros, si bien, todos parecen coincidir en su crítica al modo de proceder científico-tecnológico.

Será conveniente comenzar por plantear qué entendemos por enfoque científico-tecnológico de la educación, antes de reflexionar sobre las propuestas de la investigación- 
acción. Para ello expondré la cuestión teniendo en cuenta el desarrollo que ha tenido en España durante toda la década de los ochenta.

\section{EL ENFOQUE CIENTÍFICO-TECNOLÓGICO DE LA EDUCACIÓN}

Me limitaré a resaltar algunas de sus notas más características:

1. La educación es una parcela de la realidad humana y social susceptible de conocimiento, lo mismo que la salud, el consumo, la economía, las comunicaciones, etc.

2. El conocimiento de la educación es necesario porque sólo a partir de él es posible intervenir conscientemente y con conocimiento de causa, en su estructura, el proceso o el producto de la misma.

3. El análisis del estudio científico de la educación, al permitirnos elaborar teorías con mayor capacidad explicativa y comprensiva de los hechos educativos, se convierte en una herramienta indispensable para guiar la intervención pedagógica. En ello se justifica la muy conocida frase de que «nada hay tan útil como una buena teoría».

4. En el estudio de la educación tropezamos con una realidad polimorfa con un grado considerable de complejidad, donde el carácter relacional se da, de hecho a varios niveles, conjugándose en cada uno de ellos lo biológico, lo psicológico, lo social, lo cultural, etc., de modo que parece difícil abordarlo en su globalidad. Es por ello que, en las Ciencias de la Educación, nos encontramos con teorías científicas parciales y teorías filosóficas particulares, según el modelo antropológico que en cada caso, se propugna como deseable.

5. Por esta razón y como contrapeso del atomismo positivista, que genera un conocimiento en el que lo que interesa son las «unidades mínimas de conformación de la realidad», se propugna un método de estudio de la complejidad, que encuentra fundamento en la Teoría General de Sistemas y en la Cibernética. En este caso el hecho relacional, la interacción entre las personas, se convierte en el denominador común de cualquier acción social, como es la educativa.

6. La distinción entre los conceptos de acción y hecho va a ser medular en la propuesta científico-tecnológica, planteando el doble enfoque que se deriva de la consideración de la educación como hecho, como fenómeno que es posible describir; y en segundo lugar, de la consideración de la educación como acción que es necesario normativizar en el terreno práctico. La educación no es un hecho de la misma naturaleza que los que describen las ciencias de la naturaleza, sino que es un ejemplo claro de acción humana acerca de la cual el hombre tiene que pensar cómo actuar.

7. De aquí nace una primera propuesta científico-tecnológica acerca del conocimiento pedagógico: «Creemos que la pedagogía puede participar en la racionalidad teórica que domina la ciencia contemporánea y en la racionalidad que inspira la tecnología de nuestro tiempo» (García Carrasco, 1980, p. 10).

8. Se busca la reintegración entre la teoría y la práctica educativas, desde la referencia a la acción, ya que, como hemos visto, es posible aprovechar el estudio de la educación no sólo para aumentar nuestros conocimientos del hecho educacional, sino también para incrementar la eficacia de nuestra acción educativa. Por lo tanto, se espera que la teoría tecnológica de la acción educativa explicite aquellas secuencias de acción que permitan acercarnos a la consecución de las metas a conseguir, disminuyendo el im- 
pacto de otros factores incontrolados, entre otros, los de los propios prejuicios o supuestos previos no suficientemente contrastados del propio educador.

De manera que se produce un ciclo constante entre teoría, diseño de la práctica, acción, evaluación y vuelta a la teoría. De lo que se trata es de mejorar la práctica y la teoría, de manera que la intervención educativa se convierta, en la medida en que sea posible en intervención pedagógica, es decir, que contenga algún grado de racionalidad científica.

9. Desde esta perspectiva, la acción educativa debe seguir una secuencia parecida a la siguiente (Castillejo, 1985, p. 54; García Carrasco, 1984, p. LXX):

a) Identificación del estado inicial en el que se encuentra el sistema sobre el que se interviene.

b) La determinación de un objetivo considerado valioso.

c) Estudio de las posibilidades de intervención, en función de los objetivos, de los recursos disponibles y de las teorías que puedan ser utilizadas como recursos explicativos.

d) Diseño de la secuencia a seguir en la intervención educativa.

e) Establecimiento de criterios de evaluación capaces de relacionar el papel de las transformaciones producidas por la intervención, en el paso del estado inicial al estado final.

f) Retroalimentación sobre la teoría y sobre la práctica.

10. El resultado del conocimiento científico y tecnológico de la educación tiene sólo un carácter instrumental para la intervención educativa. Y, a la inversa, la acción educativa se convierte en la fuente que retroalimenta las teorías y los procedimientos empleados, los diseños, etc.

11. Lo que justifica la intervención es la pretensión de ayuda a la transformación del ser humano de acuerdo con algún modelo considerado como valioso. Con una determinación tan genérica, se pretende que el modelo sirva para el análisis y la implementación de modelos concretos de índole diversa, adquiriendo así las características de una metateoría. Lo cual no evita que en cualquier acción educativa esté explícita o implícitamente, o ambas cosas a la vez, la cuestión ineludible de lo axiológico, lo ético, lo político y la ideológico.

En definitiva, la perspectiva científico-tecnológica supone un curso de acción razonado que puede emplearse para los fines más diversos, con metodologías y técnicas muy distintas.

\section{LA PROPUESTA DE LA INVESTIGACIÓN-ACCIÓN}

A continuación, nos interesa reflexionar acerca de si la investigación-acción responde al modelo científico-tecnológico, si tiene rasgos distintivos y cuáles son sus contribuciones más importantes.

1. En mi opinión, la investigación-acción es una teoría acerca del hecho y de la acción educativa. No me extenderé en ello, pero al hecho educativo se lo considera como un fenómeno de carácter subjetivo, complejo, inacabado, codeterminado por la significación que le dan los protagonistas del mismo, etc. A la acción educativa se la considera como una reflexión práctica, participativa, abierta, etc. 
2. Su objetivo fundamental es el perfeccionamiento de quienes participan en cada situación educativa, la transformación y perfeccionamiento de los procesos de acción educativa y de actividad educativa. Se considera que es una investigación educativa en el sentido que no sólo se realiza sobre la educación, sino que es en sí misma educativa. En relación al logro mayor o menor de estos objetivos se puede valorar su eficacia.

3. Su objetivo secundario es la producción de conocimiento transferible de forma limitada a otras situaciones, siempre que estas guarden grados de isomorfismo elevado.

4. Se propone salvar el vacío entre la teoría y la práctica, entre la investigación y la acción. En opinión de Pérez Gómez (1992, p. 118), este distanciamiento se ha producido por tres factores:

a) Por la temática de la investigación.

b) Por la forma de producirse.

c) Por la relación de dependencia y subordinación que se ha establecido entre el investigador y el educador.

5. El educador se convierte en investigador cuando reflexiona en y sobre la acción, porque entonces construye una teoría adecuada a la singularidad del contexto en el que trabaja, y elabora un diseño que reconduce de forma continua, asentando así las bases de su propio desarrollo profesional (Pérez Gómez, 1988, p. 139).

Hay varias maneras de proceder, de actuar en educación, de acuerdo a los requisitos que se derivan de la investigación-acción, todas ellas pueden caracterizarse por compartir lo que Schon (1983) ha acuñado con el nombre de pensamiento práctico. Este pensamiento incluye tres diferentes conceptos o pasos que, a mi entender constituyen una estipulación normativa que con carácter general se ajusta a la imprevisibilidad, complejidad, singularidad, etc. de las situaciones educativas. Esta estipulación distingue:

a) Conocimiento en la acción, que es aquel que se activa para emprender cualquier acción, que es fruto de la experiencia y de la reflexión pasadas, y que muchas veces tiene un carácter implícito que se manifiesta en rutinas y esquemas semiautomáticos de comportamiento.

b) Reflexión en la acción, que es el tipo de reflexión que realizamos mientras actuamos, carente de sistematicidad y distanciamiento, pero con la riqueza de la inmediatez y de la presencia ante el suceso. Es un primer espacio de confrontación entre los esquemas teóricos, teorías implícitas y creencias con la realidad singular en la que se está.

c) Reflexión sobre la acción y sobre la reflexión en la acción, que es una reflexión más distante y sistemática sobre el conjunto de elementos que han intervenido en la acción: diagnóstico de la situación, fijación de metas, elección de medios, la propia intervención, las teorías empleadas para interpretar la situación, etc.

Podríamos decir que el educador intenta clarificar lo que ha ocurrido en la situación de enseñanza/aprendizaje, intentando desvelar su sentido reflexionando sobre lo ocurrido, sobre lo realizado y sobre las razones explícitas e implícitas.

Este apartado es muy importante para determinar si la investigación-acción es un caso de pensamiento científico-tecnológico, ya que en mi opinión representa una estipulación de un procedimiento o un conjunto de normas o estrategias, o como se las quiera denominar, que se consideran como requisitos que ha de cumplir el modo de actuar del educador para poder conseguir un cambio de estado, una transformación. Transformación que en este caso se pretende múltiple, ya que afecta al contexto de significados 
compartidos (cultura del grupo), a los educadores, a los alumnos, a la propia teoría o repertorio de conocimientos y a procedimientos previos a la acción.

¿Se puede considerar el pensamiento práctico como una competencia o un conjunto de competencias complejas que ha de adquirir el educador? ¿Están derivadas esas competencias de un conocimiento científico previo acerca de lo que es la acción educativa? Si es así, no parece haber gran diferencia entre la investigación-acción y el modelo científico-tecnológico tal y como ha venido desarrollándose en los últimos años.

Así, por ejemplo, se define el pensamiento práctico como «una compleja competencia de carácter holístico» y se añade que «lo que se intenta formar a través del currículum profesional es la capacidad de intervenir de manera competente en situaciones divergentes, y que esta capacidad o pensamiento práctico, es un conjunto idiosincrásico, (...) de conocimientos, capacidades, teorías, creencias y actitudes» (Pérez Gómez, 1988, p. 145). Parece que lo que se quiere destacar es el hecho de que ese repertorio es idiosincrásico, es decir, propio y particular de cada educador en el contexto de la intervención. Pero sin entrar a discutir esa cualidad, lo que me importa destacar es que se trata de un modo de proceder tecnológico. El hecho de partir de la práctica, aún en el supuesto de que se prescinda de cualquier teoría más o menos científica, no le resta a este modo de proceder un carácter tecnológico.

6. Lo que la I.A. niega fundamentalmente es que pueda considerarse la acción del educador como una actividad prioritaria y exclusivamente tecnológica, porque debe ser más bien una actividad reflexiva y artística (Cfr. Pérez Gómez, 1988, p. 133), en la que secundariamente tienen cabida algunas acciones tecnificadas.

El carácter reflexivo y artístico viene exigido por las características de los fenómenos prácticos, siendo la educación un caso de ellos: complejidad, incertidumbre, inestabilidad, singularidad y conflicto de valores (Schon, 1983). Y parece que lo que se pretende resaltar con esta metáfora del educador como práctico-reflexivo o como artista es que las situaciones con las que se enfrenta no pueden resolverse con la aplicación mecánica de reglas, técnicas o procedimientos estandarizados, convertidos en rutinas aplicables a cualquier situación educativa. Pero la cuestión que me interesa plantear aquí es, precisamente, si el educador para actuar realiza un proceso de reflexión que podamos caracterizar como tecnológico, o si es otra cosa. Desde luego, cuando aplica secuencias de pasos estandarizadas sin ningún tipo de reflexión antes, durante y después del proceso educativo, no podemos decir que tenga un pensamiento tecnológico. En este caso no sería ni pensamiento. Pero si cuando realiza diagnósticos de la situación, cuando observa, cuando plantea objetivos, cuando trabaja con metodologías y recursos, cuando aplica conocimientos, cuando evalúa los procesos y los contextos, etc. Evidentemente, como muchas de las situaciones pueden ser impredictibles, tendrá que buscar soluciones sobre la marcha que después matizará reflexivamente, pero hasta eso puede ser entrenado, precisamente con muchos de los procedimientos que defiende la I.A. Cuando el educador «construye una teoría adecuada a la singular situación de su escenario y elabora en su interacción con la situación un diseño flexible de enfoque progresivo que experimenta y reconduce de forma continua» (Pérez Gómez, 1988, p. 139) se comporta como un tecnólogo que pretende resolver problemas que le plantea la práctica educativa.

7. Pero hay una diferencia que los partidarios de la dicotomía se interesan en remarcar. Me refiero a la poca importancia que le conceden a los aportes teóricos y metodológicos previos a la acción, por entender que tienen una capacidad de transferencia 
muy limitada. Sin embargo, esta falta de consideración no parece lógica puesto que el conocimiento lo mejor fundado posible es una buena forma de enriquecer el pensamiento práctico del educador. Otra cosa será discutir qué teorías o procedimientos son más aconsejables, o se ajustan más a las características de la realidad sobre la que se va a actuar. Así por ejemplo, la I.A. se define como una nueva «epistemología de la práctica», como una concepción diferente de la relación entre conocimiento y acción, entre teoría y práctica», capaz de preparar al educador para actuar en situaciones prácticas divergentes (Cfr. Ibid., p. 142). ¿No es acaso la I.A., así definida, una teoría acerca de la educación que se postula como capaz de fundamentar la acción educativa? Si es así, ¿por qué, entonces se niega la utilidad de aportes teóricos y metodológicos previos a la práctica concreta de un individuo singular? ¿No es acaso la propia I.A. una teoría acerca de la acción de carácter externo y que se propugna como aplicable por su adecuación al objeto de estudio, con carácter global para cualquier situación educativa? Si la respuesta fuera afirmativa, la cuestión se reduciría a saber qué tipo de tecnología es.

8. Una vez definido el pensamiento práctico como modo más adecuado de proceder en educación y una vez descrito en qué consiste investigar en la acción, ambos se convierten en artefactos conceptuales y metodológicos que pueden enseñarse y aprenderse, ya que no parece congruente que pueda realizarse conscientemente lo que se desconoce. Y esta tarea, hay que reconocer que la deben hacer, como de hecho la están haciendo los teóricos de este enfoque. Tal y como ha ocurrido con el modelo que denominan tecnológico.

9. Como cualquier tecnología, la investigación-acción propone un repertorio de procedimientos metodológicos de recogida de datos para interpretar la realidad educativa y el significado que adquiere para sus protagonistas: la observación participante, la entrevista, la triangulación, los diarios de campo, etc. Casi todos ellos de carácter etnográfico porque tal investigación debe hacerse en el contexto. El conjunto de todos ellos producen un conocimiento ajustado al objeto de estudio que permite comprender la práctica para transformarla (Ibid., 1992, p. 129). El conocimiento teórico obtenido de otras situaciones puede ser utilizado, pero sólo como instrumento que posibilita la reflexión sobre la práctica específica.

10. Se señala como limitación de la racionalidad tecnológica su pretensión de objetividad, entendida como una característica de aquellas interpretaciones supuestamente libres de condicionantes culturales y contextuales, capaces de ser generalizadas y transferidas a cualquier situación. A esta objeción se podría contestar diciendo que no toda tecnología, por el hecho de serlo, ha de partir de supuestos estrictamente positivistas a la manera de las ciencias físicas. A una tecnología se la puede adjetivar, pero no se la define por el soporte epistemológico en el que se fundamenta. Por el contrario, hoy está aceptada la idea de que el conocimiento científico es una aproximación progresiva del conocimiento a la realidad y también un proceso de producción y transformación de nueva realidad, por cuanto los resultados de la ciencia y la tecnología, tienen sus claros reflejos en la transformación de la vida social. El conocimiento científico, según afirma Bunge (1981), implica la construcción de teorías que constituyen una representación parcial de la realidad. Por esta razón, las teorías fracasan tarde o temprano, en beneficio de otras que representan mejor la realidad. Muy diferentes autores, coinciden en la idea de que, en sentido estricto, «son muy pocos los sectores que poseen un desarrollo cien- 
tífico que haya permitido tal tipo de construcciones (en educación)» (García Carrasco, 1984, p. XVII).

Cuando hablamos de ciencia al referimos a la educación o a lo socio-humano en general, lo que tratamos es de superar el sentido común, la mera opinión o lo que son las creencias. La epistemología actual no permite afirmar que el método científico sea una secuencia de pasos de obligatorio y estricto cumplimiento en todos los casos, independientemente de la naturaleza de lo que se pretende conocer (Touriñán, 1987, p. 190). Por la poderosa influencia, ejercida a lo largo de la historia de la ciencia, de los modelos de las ciencias físico-naturales, se ha pretendido juzgar la validez del conocimiento de todo tipo de realidad aplicando los criterios empleados en esos modelos. Sin embargo, es generalmente aceptado que no puede haber un método predeterminado, sino que éste dependerá, en cada caso, de la estructura que presente la realidad que se investiga. Quizás uno de los requisitos más importantes exigible a cualquier metodología es el que sea explícita en los pasos seguidos, en los supuestos en los que se basa y, por lo tanto, que permita la crítica pública de quienes trabajan en un campo de conocimiento determinado. De este modo se garantiza que otros investigadores sepan el camino que se ha seguido y puedan repetirlo con el fin de depurar los errores y ratificar los resultados (Cfr. Esteve, 1986, p. 77). El conocimiento científico, frente al conocimiento vulgar, debe poder evaluarse, y para ello, los investigadores o las corrientes que los aglutinan, tienen que explicitar su propuesta sobre él y los límites de validez que asumen (Ladriere, 1977, p. 115).

Lo objetivo, en sus expresiones más abiertas, se puede identificar con lo público, es decir, con lo abierto a todo aquel que posea información pertinente y pueda explicar la forma en que la ha producido y el modo en que la justifica (Cfr. Touriñán, 1987, p. 122).

De todas formas, sí quisiera resaltar que lo que el científico está obligado a evitar es la falta deliberada de objetividad en las diferentes fases de la investigación, pues si bien no puede evitar partir de unos supuestos previos que limitan sus resultados, lo que no debe tener es carencia deliberada de veracidad, o intención de falsear los resultados que obtiene.

La I.A. basándose en sus propios supuestos y ateniéndose a las características que considera que caracterizan la realidad educativa prescribe unos métodos y desecha otros, o bien sin llegar a desecharlos, los considera como menos pertinentes. Y ello en función de unas características que se predican de la realidad a estudiar. Aquellos que considera menos útiles son los experimentales.

11. Otra objeción que suele hacerse al modo de proceder tecnológico es su presunta incompatibilidad con el caso singular. Tampoco esto es cierto salvo en el caso de tecnologías extremadamente positivistas. Dentro de los planteamientos tecnológicos se acepta que en educación no siempre es posible la creación de generalizaciones nomológicas ni probabilísticas, es decir, no siempre se pueden explicar los casos concretos en base a una ley general o de alta probabilidad. El problema de lo ideográfico surge cuando nos encontramos con condiciones que sólo se dan en el caso concreto que queremos explicar y que, por lo tanto, sólo le son aplicables a él mismo (Cfr. Vázquez Gómez, 1980, pp. 44-46 y Touriñán, 1987, p. 206).

12. Otro tanto ocurre cuando se quiere pretender que cualquier intento por predecir y controlar los procesos niega de raíz la posibilidad de la libertad (Cfr. Pérez Gómez, 1992, p. 122). Con la misma gratuidad se podría decir justamente lo contrario, ya que el control puede ejercerse sobre los procesos que interfieren la capacidad crítica y la auto- 
nomía, tanto como los que las posibilitan. La racionalidad tecnológica no presupone un tipo determinado de fines educativos, puesto que éstos no se pueden determinar sino por decisiones axiológicas, políticas, etc. Aunque desde mi punto de vista, si hablamos de procesos educativos, hablamos de desarrollo de la autonomía, en caso contrario deberíamos emplear el término de procesos configurativos u otros. Al decir ésto, no quiero sino indicar que el problema de los fines es previo a lo tecnológico y que requiere otro tipo de reflexión, no que el educador sea sólo tecnólogo y que deba despreocuparse de lo axiológico, ni de lo político.

La investigación-acción al producir conocimiento y suponer una intervención en la educación que incluso trata de llegar a desvelar los significados que los hechos tienen para los participantes, y por tener como objetivo la transformación de la práctica, requiere igualmente la capacidad de control del mayor número posible de variables y de significados, incluso los de las propias teorías implícitas. Siempre que hay control de los procesos se puede caer en la tentación de emplearlo para la manipulación o el adoctrinamiento, pero si no hay intervención intencionalmente educativa, el sujeto queda expuesto al influjo de los procesos de socialización, al aprendizaje de las conductas sociales de su grupo, y en ello coincide tanto la investigación-acción, como la propuesta tecnológica.

13. El último aspecto que voy a tratar en estas reflexiones es el de la relación jerárquica entre el educador con respecto al investigador o a las disposiciones administrativas de los políticos. Es verdad que existe una distancia entre quienes producen conocimiento pedagógico y los educadores que supuestamente han de aplicarlo, pero de aquí no se deduce que se deba al pretendido predominio de la racionalidad tecnológica. En este sentido, no creo que la elaboración teórica de la investigación-acción garantice que ésta sea realizada mayoritariamente por los educadores en los diferentes ámbitos educativos. Si lo hacen será porque tienen esa inquietud y porque se les incentiva para ello. Por otra parte, nadie puede producir todo el conocimiento ni los métodos que precisa en su trabajo, es necesaria la colaboración de muchos.

Este asunto tiene mucho que ver con el tipo de educador que se pretenda lograr, con la formación que se le facilite, con las prácticas en las que deba intervenir, y con el estatus que se le quiera dar. Creo que es asumible por todos que el educador debe tener una buena preparación pedagógica de base que le permita una reflexión sistemática y crítica sobre su práctica, sobre las condiciones en las que ejerce su trabajo, sobre su rol, etc. (Villar Angulo, 1992, p. 9). Pero para lograrlo, no encuentro mejor forma que incrementar su preparación científico-tecnológica, porque si no corremos el riesgo de hacer reflexiones e investigaciones que, por prescindir de aspectos ya trabajados con anterioridad, sean menos fructíferas para la propia formación y para la transformación de la realidad educativa. También se puede ser un buen o un mal artista según el dominio de las reglas y técnicas con las que se construye cualquier arte, pongamos como ejemplo el de la interpretación teatral.

Como conclusión más general quisiera terminar reconociendo la importancia de la propuesta de la investigación-acción, la utilidad de la metodología cualitativa que propone y el papel del educador como intelectual que participa en el diseño de su práctica y que reflexiona críticamente sobre ella (un tecnólogo crítico). Pero nada de ello veo que contradiga la necesidad de un enfoque científico tecnológico, independientemente de cuales sean sus supuestos, no encontrando razones que nieguen la posibilidad de considerar a la propia investigación-acción como un modelo científico-tecnológico, de corte 
socio-crítico en sus premisas epistemológicas y preferentemente cualitativo en su soporte metodológico.

\section{BIBLIOGRAFÍA}

Bunge, M. (1981). Teoría y realidad. Ariel, Barcelona, $3^{\text {a }}$ ed.

CASTILlejo, J. L. (1985). «Sugerencias para una teoría de la ciencia pedagógica». En P. AzNAR, y otros. Conceptos y propuestas (II). Nau Llibres, Valencia.

Colom, A. J. (1982). Teoría y metateoría de la educación. Trillas, México.

Esteve, J. M. (1986). «Respuesta de la pedagogía frente a los criterios de demarcación de la ciencia». En Analisi Pedagogica, 1, Roma, pp. 73-106.

ETXEBERRía BALERDI, F. (Dir) (1989). Pedagogía social y educación no escolar, Universidad del País Vasco, San Sebastián.

Fullat, O. (1984). Verdades y trampas de la Pedagogía. CEAC, Barcelona.

GARCía CARRASCO, J. (1980). Aproximación al estudio de la estructura del acto pedagógico. Universidad Pontificia, Salamanca.

García CARRAsCo, J. (1984). Teoría de la educación, Anaya, Madrid.

GARCÍA CARRASCO, J. (1987). «Fracaso escolar e innovación educativa». En F. ETXEBERRÍA (ed.). El fracaso de la escuela. Erein, San Sebastián, pp. 41-54.

García Carrasco, J.(1988). «Planteamiento tecnológico de la institución docente». En A. Villa (ed.). Perspectivas y problemas de la función docente, pp. 314-326.

LADRIERE, J. (1977). El reto de la racionalidad, Sígueme, Salamanca.

PALAZÓn Romero, F. (1992). «La investigación-acción como metodología puente entre la educación de adultos y el desarrollo comunitario». Pedagogía Social, nº 7, pp. 51-61.

PÉREZ GómEZ, A. (1988). «El pensamiento práctico del profesor: implicaciones en la formación del profesorado». En A. Villa (ed.). Perspectivas y problemas de la función docente, pp. 128148.

PÉRez Gómez, A. (1992). «Comprender la enseñanza en la escuela. Modelos metodológicos de investigación educativa». En J. GIMENo y A. PÉREZ Comprender y transformar la enseñanza, Morata, Madrid, pp. 115-136.

SÁEz CARreras, J. (1992). «Los educadores sociales ¿tecnólogos o intelectuales?». Pedagogía Social, $\mathrm{n}^{\circ} 7$, pp. 179-189.

SARRAMONA, J. (1990). Tecnología educativa (una valoración crítica), CEAC, Barcelona.

SCHON, D. (1983). The reflective practitioner, Basic Books, Nueva York.

Touriñán, J. M. (1987). Teoría de la educación, Anaya, Madrid.

TOURIÑÁN, J. M. (1991). «Conocimiento de la educación y función pedagógica: el sentido de la competencia profesional». En Rev. Teoría de la Educación, III, pp. 13-27.

VÁzQuEz GómEZ, G. (1980). «Unidad, autonomía y normatividad en la investigación pedagógica. Consecuencias para la formación de profesores». SEP. La investigación pedagógica y la formación de profesores, VII Congreso Nacional de Pedagogía, Tomo II, pp. 39-61. 
VÁZQUEZ GÓMEZ, G. (1987). «El modelo de investigación-acción en el currículum». En SARRAMONA, J. (Ed.). Currículum y educación, CEAC, Barcelona.

Vera Vila, J. (1989). Proyecto docente presentado al concurso de la plaza de Profesor Titular de Universidad. Málaga.

Villar Angulo, L. M. (1988). «Reflexiones en y sobre la acción de profesores de EGB en ejercicio en situaciones interactivas de clase». En A. VILla (Ed.). Perspectivas y problemas de la función docente, pp. 149-174.

Villar Angulo, L. M. (1992). «Teorías implícitas de los profesores sobre el cambio educativo». Ponencia presentada al VII Congreso de la AIRPE, Salamanca, 22-25 de abril. 